\title{
Reflexiones situadas sobre la extensión universitaria, la vinculación territorial y la integralidad
}

\section{Situated reflections on university community outreach programs, territorial linkage and integrality}

\section{Silvina Gómez}

https://orcid.org/0000-0001-9902-8879

silvinabgomez@gmail.com

Facultad de Ciencias Económicas

| UNLP | Argentina

\author{
Elisabet Rossi \\ https://orcid.org/0000-0001-9621-0827 \\ elisabetrossi@gmail.com \\ Facultad de Ciencias Económicas \\ | UNLP | Argentina
}

\footnotetext{
Gonzalo Márquez

https://orcid.org/0000-0001-7378-836X

cosmegjm@gmail.com

Facultad de Ciencias Naturales y

Museo | UNLP | Argentina
}

\section{RESUMEN}

En el siguiente artículo se plasmarán algunas reflexiones situadas en vinculación con una práctica de extensión llevada a cabo en los últimos 8 años. Durante los años de trabajo en el territorio, se desarrollaron de manera simultánea un conjunto de procesos integrados que reflejan "el hacer" de la extensión a partir de una relación sinérgica universidad-sociedad: el acceso al territorio, el establecimiento de lazos, la creciente institucionalización de la extensión universitaria como contexto de la intervención llevada adelante, la propia reconfiguración en la comprensión del territorio y de la relación universidad- sociedad, y el aprendizaje situado tanto de las y los docentes como de las y los estudiantes, y la consiguiente reflexión acerca de estos procesos. Finalmente la toma de conciencia y posición acerca de la centralidad de la extensión en una renovada forma de entender la producción de conocimientos, más democrática y respetuosa de los conocimientos co-producidos con otros y otras fuera de los muros de la universidad.

\section{ABSTRACT}

The following article will reflect some situated considerations in connection with an Extension practice carried out along the last 8 years. During the time worked on the field, a set of integrated processes were simultaneously developed, reflecting the "making" on extension programs from a synergistic university-society relationship: the access to the territory, the establishment of social ties, the growing institutionalization of university extension as a context for the intervention we carried out, the reconfiguration of the understanding of the territory and the university-society relationship, and the situated learning of both teachers and students, as well as the consequent reflection about these processes. Finally, the awareness and position about the centrality of the extension in a renewed way of understanding the production of knowledge, as a more democratic and respectful way of understanding the co-produced knowledge with others outside the walls of the university.

\section{PALABRAS CLAVE}

Extensión Universitaria, Integralidad,

Turismo,

Patrimonio,

Economía social

\section{KEY WORDS}

University Extension Projects, Integrality,

Tourism,

Heritage,

Social and Solidarity Economy 


\section{INTRODUCCIÓN}

En el presente artículo se propone reflexionar sobre la experiencia territorial adquirida desde el año 2012 al presente con su continuidad mediante el desarrollo conjunto del programa de extensión universitaria "Turismo, patrimonio y desarrollo en el periurbano platense" y del proyecto de extensión universitaria específica "Senderos turísticos recreativos en el periurbano platense". En dicha experiencia de extensión se buscó construir un vínculo entre las y los productores e integrantes de las comunidades locales y nuestro equipo extensionista, con el objetivo de trabajar articuladamente para poner en juego las lógicas propias universitarias y las lógicas de las y los actores territoriales. Articulación que permitió profundizar alianzas con los grupos y organizaciones sociales y productivas, $y$ dar visibilidad a sus problemáticas, tanto sociales como culturales, políticas, económicas y ambientales '

Durante los años de trabajo en el territorio, se desarrollaron de manera simultánea un conjunto de procesos integrados que reflejan "el hacer" de la extensión a partir de una relación sinérgica universidad-sociedad que, como práctica colectiva y dialógica, pone en juego a diversos actores comunitarios, institucionales y universitarios, en pos de encontrar las vías que provean soluciones a las problemáticas anteriormente mencionadas.

Históricamente se pensó la extensión universitaria como la provisión de conocimientos de la universidad hacia la sociedad, el derrame y la iluminación de quienes poseen el saber hacia quienes no lo poseen. En las últimas décadas esta práctica se ha repensado, poniéndose en evidencia la necesidad de reconocer que aquellos que eran considerados "beneficiarios" de la extensión, son protagonistas de sus 
acciones con conocimientos y saberes teóricos y prácticos. Trabajar la extensión desde esta nueva concepción de la práctica universitaria nos lleva a la integración de procesos que articulan la extensión, la docencia y la investigación.

La construcción de los espacios y actividades durante nuestra actividad extensionista, se llevó a cabo de manera horizontal entre las y los diferentes actores y sectores del periurbano platense, con el propósito de alcanzar, mediante el turismo recreativo, el desarrollo comunitario. Dichas prácticas permitieron a las y los estudiantes /extensionistas el contacto directo con las problemáticas de las y los productores flori-hortícolas y sus saberes, lo cual propició la construcción conjunta y apropiación de estos nuevos conocimientos, basados en el diálogo de saberes académicos y populares. A su vez, las y los productores fueron los protagonistas de dicho proceso con la consecuente apropiación de los objetivos del proyecto.

De manera simultánea, la universidad como institución pone en juego su función social y política, en contacto permanente con el contexto, enriqueciendo sus prácticas y conocimientos, los cuales son adquiridos por las y los docentes, y las y los estudiantes universitarios, como nuevos modos de comprender la compleja dinámica social. De este modo, así como la práctica universitaria transforma la realidad del territorio, la participación activa de las y los actores sociales en dicha práctica, transforma las estructuras de la propia universidad, con la incorporación de nuevos conocimientos y la democratización en la producción de los mismos.

Por lo expuesto en los párrafos anteriores, es de nuestro interés poner de manifiesto la importancia que ha tenido la evolución del concepto "extensión" en la universidad argentina y como se ha convertido en una herramienta fundamental para el desarrollo territorial, a partir de una relación democrática y sinérgica entre el ámbito académico y el productivo. Teniendo en cuenta estos desarrollos, nuestra intención es reflexionar sobre la centralidad de nuestra práctica extensionista en las actividades docentes y de investigación que llevamos a cabo, comprendiendo que las mismas se interrelacionan profundamente, y que las lógicas de la intervención territorial transforman no sólo el conocimiento que tenemos del territorio sino la forma de concebir la producción de conocimientos.

Teniendo en cuenta estos desarrollos, nuestra intención es reflexionar sobre la centralidad de nuestra práctica extensionista en las actividades docentes y de investigación que llevamos a cabo, comprendiendo que las mismas se interrelacionan profundamente, y que las lógicas de la intervención territorial transforman no sólo el conocimiento que tenemos del territorio sino la forma de concebir la producción de conocimientos. 
A continuación haremos un breve recorrido por el contexto institucional de nuestra práctica extensionista y los cambios que se han desarrollado en este ámbito en los últimos 15 años. Luego veremos cómo el trabajo territorial atraviesa las fronteras de la teoría y fuerza el repensar las categorías con las cuales trabajamos en los ámbitos docentes y de investigación, por último compartiremos algunas reflexiones sobre los procesos de co-construcción de conocimientos realizados a través del trabajo territorial.

\section{CONTEXTO INSTITUCIONAL.}

A partir de 1918, la universidad argentina adopta el modelo "reformista" (Carli, 2008), donde la Extensión Universitaria pasa a ser "función central", considerando que la misma consiste en una devolución a la sociedad, por parte del estudiante, de la posibilidad de estudiar que ésta le brindó.

Como señala Drago retomando a Tünnermann Bernheim "el movimiento (reformista) agregó un nuevo cometido a la universidad, capaz de vincularla más estrechamente con la sociedad y sus problemas, de volcarla hacia su pueblo. Acorde con esta aspiración, la Reforma incorporó la extensión universitaria y la difusión cultural entre las tareas normales de la universidad latinoamericana." (Drago, 2000)

Las actividades englobadas dentro de lo que se denominó Extensión Universitaria, implicaban una "bajada" de conocimientos desde quienes poseían los conocimientos académicos y científicos, a quienes carecían de ellos, siendo de esta forma una transmisión unidireccional de saberes doctos hacia "la sociedad".

No obstante, esta función primordial de la Universidad Pública no fue desarrollada de manera formal y permanente por todo el sistema universitario. Con la crisis social y económica del año 2001, proliferaron las organizaciones territoriales y la universidad comenzó a focalizar la mirada en ellas. A partir del año 2002 comenzó una política activa desde la Secretaría de Políticas Universitarias de la Nación (SPU) para promover la actividad extensionista, lo que devino en la creación de ámbitos formales y jornadas de encuentro académico, que confluyeron en la creación de la red REXUNI del Consejo Interuniversitario Nacional (CIN), que funciona como un espacio de coordinación para problemáticas comunes. Sin embargo, aun con esta activación de la actividad extensionista, como señala Drago (2016), la misma aún era y es considerada por los propios involucrados como una función subsidiaria y desjerarquizada.

También a partir de esta época, se dio un proceso de democratización del acceso universitario "...impulsando el ejercicio efectivo del derecho a la educación superior por parte de las y los trabajadores de este campo, más aún de quienes participan en iniciativas de la eco- 
nomía social emergente tales como, entre otros, trabajadores vinculados a la economía popular, emprendedores de ferias y mercados de la Economía Social y Solidaria (ESS), integrantes de cooperativas de trabajo y empresas recuperadas, recicladores urbanos, productores de la agricultura familiar o promotores territoriales de microcrédito." (Pastore, 2015)

En la transformación de esta forma de concebir a la sociedad, a la universidad y las vinculaciones entre ellas, tuvo fuerte influencia Paulo Freire, con sus críticas a la educación clásica como sistema bancario y la necesidad de concebir educación popular como educación liberadora, así como sus críticas a la "extensión" en contraposición a la comunicación. "Educar y educarse, en la práctica de la libertad, no es extender algo desde la "sede del saber" hasta la "sede de la ignorancia", para salvar, con este saber, a los que habitan en aquélla. Al contrario, educar y educarse, en la práctica de la libertad, es tarea de aquellos que saben que poco saben, por esto saben que saben algo, y pueden así, llegar a saber más, en diálogo con aquellos que, casi siempre, piensan que nada saben, para que éstos, transformando su pensar que nada saben en pensar que poco saben, puedan igualmente saber más." (Tünnermann Bernheim, 2000:8)

Estas ideas han permeado, aunque de modo desigual, los cristales de las universidades, concebidas como torres del conocimiento, posibilitando nuevas formas de relación universidad-comunidad, y dando lugar a visiones novedosas como la "extensión a la inversa" que, como cita Pastore, "... promueve una ciudadanía universitaria activa para las y los actores territoriales y populares con que se vincula, oponiéndose a considerarlos como "objeto" de estudio, asistencia o transferencia; antes bien, buscando garantizar su condición como sujetos plenos de derecho, capacidades y responsabilidades." (2015)

Abonando en este sentido, la generación de líneas de financiamiento para los programas de extensión, los cuales son las verdaderas herramientas de acción en el territorio, entre los que podemos nombrar a los Programas Nacionales de Voluntariado Universitario, Educación Solidaria y Universidad Argentina, han potenciado la participación de la comunidad universitaria en estas actividades, lo que redunda un mayor compromiso con un modelo de universidad más comprometido con su entorno.

En la Universidad Nacional de La Plata (UNLP), con la creación del Consejo Social en el año 2010, producto de una política activa a nivel nacional amparada en el artículo 56 de la Ley de Educación Superior (LES) que promueve estos espacios formales en las Universidades Públicas, se cuenta con un ámbito institucional adecuado para el encuentro de las y los extensionistas, los cuales diseñan líneas activas de problemáticas a resolver en la región, tanto en clave de extensión como para la investigación y la transferencia. La composición de este 
cuerpo es heterogénea en miradas y democrática en funcionamiento, ya que nuclea con voz y voto a diversos actores sociales, gremiales, gubernamentales e internos de la Universidad. Otra estrategia de la UNLP para generar una política de gestión situada en el territorio fue la creación de los Centros Comunitarios de Extensión Universitaria (CCEU), como espacios co-gestionados para la problematización de lo que ocurre en los diferentes barrios donde se encuentran estos dispositivos, a partir de las voces de todos las y los actores, descentralizando la actividad extensionista.

Las Universidades cumplen un rol activo como vehiculizadoras de propuestas consensuadas a problemas sociales, productivos o culturales entre los diversos actores territoriales, a través de sus propuestas de extensión. De esta forma, intervienen para lograr el encuentro entre los distintos espacios gubernamentales, no gubernamentales y comunitarios, sin arrogarse ni detentar las indelegables funciones básicas que debe cumplir el Estado.

\section{Las Universidades cumplen un rol activo como vehiculizado- ras de propuestas consensuadas a problemas sociales, pro- ductivos o culturales entre los diversos actores territoriales, a través de sus propuestas de extensión.}

En base a los tres ítems planteados anteriormente, que los podemos resumir en una política activa para el fomento de la extensión por parte del Ministerio de Educación de la Nación, se han generado diversas herramientas de financiamiento para el desarrollo de dichas actividades y un marco institucional en la UNLP, que han favorecido el trabajo territorial de los grupos extensionistas y han permitido realizar gran cantidad de experiencias en el periurbano platense. Las mismas, se han podido sostener en el tiempo gracias a estas condiciones novedosas y favorables, que generaron espacios nunca antes vistos en la región. Enmarcamos nuestro proyecto en dos de los ejes generales planteados por el Consejo Social de la UNLP, producto del intercambio de visiones y diagnósticos generados en los espacios horizontales creados para este fin: Sistema Productivo Regional y Condiciones Laborales; y Cultura, Diversidad, Pueblos Originarios y Derechos Humanos. De esta manera logramos insertarnos en la estrategia global de la Secretaría de Extensión de la UNLP, aportando este trabajo en el periurbano como insumo permanente para fortalecer la estrategia de inserción de la Universidad en esta área semi-rural del partido de región.

A partir de los objetivos planteados por las autoridades de la UNLP, que priorizan a la extensión universitaria en relación al fortalecimiento de la red de inclusión social, y teniendo en cuenta el rol articulador de 
la casa de estudios, el proyecto tuvo como objetivo principal la construcción patrimonial para el desarrollo de un sendero turístico rural en zonas periurbanas de la ciudad de La Plata. La persecución de dicho objetivo nos llevó durante estos años a interactuar con distintas instituciones de la comunidad, articulando con organizaciones gubernamentales y sociales con presencia en la zona de trabajo, probando una variedad de estrategias de acercamiento, de trabajo en equipo, de comunicación, entre otras.

\section{DE LOS PROCESOS TERRITORIALES Y DE LAS ESTRATEGIAS IMPLE- MENTADAS DESDE LA EXTENSIÓN UNIVERSITARIA}

Para abordar los procesos territoriales que desarrollamos desde la extensión universitaria, consideramos poner en juego los conceptos que atravesaron a dicha práctica, ya que estamos convencidos que no hay práctica de extensión que no se encuentre atravesada por un marco conceptual, político e ideológico, desde el cual se mira y se interviene. Territorio, patrimonio, identidades sociales, Economía Social y Solidaria (ESS) son los conceptos que, desde una mirada compleja y sistémica, sirven como herramientas para este abordaje y la continua reflexión acerca del mismo. En este sentido la integralidad no puede ser pensada sólo desde la reflexión teórico conceptual, sino como algo que se hace y se recrea desde las prácticas.

Para abordar los procesos territoriales que desarrollamos desde la extensión universitaria, consideramos poner en juego los conceptos que atravesaron a dicha práctica, ya que estamos convencidos que no hay práctica de extensión que no se encuentre atravesada por un marco conceptual, político e ideológico, desde el cual se mira y se interviene.

Se requiere reconocer la complejidad del territorio, para entender la complejidad del desarrollo turístico en cuanto fenómeno social, económico, cultural, ambiental y los reclamos para que el mismo sea sustentable. Reorganizar el territorio considerándolo como espacio para el uso turístico, conlleva re-valorizar y re-funcionalizar los lugares porque se producen nuevas relaciones entre el sistema de objetos y acciones en dichos espacios. (Santos, 1996)

Frente a lo expuesto, abordar la complejidad implica un enfoque sistémico que pueda proporcionar una perspectiva metodológica que permita reflexionar y analizar los sistemas abiertos. (Gallopín, 2003) Tal es el caso de los complejos espacios turísticos y recreativos, que poseen una estructura interna constituida por elementos naturales y sociales, 
acciones socioeconómicas, culturales y políticas que se interrelacionan de tal forma que conforman un sistema complejo e indisociable. Rolando García (2006) establece que, la complejidad está asociada con la imposibilidad de considerar aspectos particulares de un fenómeno, es decir, debe ser considerada no solo por la diversidad de las partes que constituyen los procesos o fenómenos de la realidad, sino además y, sobre todo, por la interdefinibilidad y mutua dependencia de las funciones que estas partes desempeñan dentro de una totalidad.

En este marco, partimos de pensar conceptualmente al turismo como un fenómeno social complejo, con múltiples facetas que involucran la esfera de lo económico, lo social-cultural y lo ambiental, donde interactúan diversos sujetos, tales como intermediarios, comunidad de origen y destino. Como señala Bertoncello (2002) el incorporar a estos sujetos, sus contextos e intenciones específicas, nos obliga a pensar al turismo en el marco de las características y dinámicas sociales específicas en las cuales el mismo se lleva a cabo, y en las cuales cobra especificidad y sentido.

El turismo es comúnmente considerado una potencial fuente de desarrollo y de mejoría de la calidad de vida de las poblaciones. Coincidimos que esta es una posibilidad, siempre y cuando se desarrolle reflexivamente y en conjunto con las poblaciones locales. Así el turismo invita a reflexionar sobre las y los actores sociales con quienes trabaja y las condiciones de vida y trabajo que éstos tienen, sus intereses, los discursos que los nombran y las políticas destinadas al sector.

Lo que el turismo muestra suele ser aquello valorado por las comunidades o por el mismo turismo, por su unicidad, su exotismo, su grandeza, su sentido histórico entre otras razones. Durante mucho tiempo prevaleció una mirada material acerca del patrimonio, que lo visualizaba casi exclusivamente en las grandes obras arquitectónicas, históricas y artísticas, que exaltan los valores de un pasado común y contribuyen a formar una idea de nación por parte del poder político y económico. “Elementos elegidos por el Estado como parte de la implementación práctica de visiones nostálgicas del pasado... símbolos de exaltación y celebración de una supuesta unidad política de la nación". (Arantes, 1997). Este es el que se denomina patrimonio institucionalizado. Aquel que se muestra como Patrimonio, con mayúsculas, que se cristaliza para conservarlo en la eternidad, y que se supone como "el único posible" (Delgado, 2006). Un patrimonio que representaría a toda la sociedad de la que surge, licuando de esa forma la heterogeneidad de la misma e invisibilizando las operaciones a través de las cuales, sectores hegemónicos logran establecer su patrimonio como el patrimonio de todos.

Del mismo modo, el patrimonio inmaterial que generalmente se visibiliza y pone en valor, también responde a las lógicas que predominan en la definición del patrimonio material: la de la pérdida y ne- 
cesidad de eternizar; la valorización de lo "excepcional" como opuesto a lo cotidiano; la descontextualización de las prácticas sociales que lo traen a la vida y lo resignifican, y la objetivación del bien cultural, volviendolo unívoco e incuestionable (Lacarrieu, 2010).

En contraposición al patrimonio institucionalizado, encontramos el patrimonio colectivo, el patrimonio no reconocido como tal, y por ende no gestionado, no rememorado. Lacarrieu advierte que lo popular, tiende a ser pensado como lo folk, como la antípoda de lo moderno, lo intelectual y lo racional, y por ello como opuesto también al progreso. Estas manifestaciones culturales no han sido valoradas en el proyecto nacional, en tanto representaban aquello que debía suprimirse y superarse en el camino hacia la modernidad. Lacarrieu, citando a Zubieta, dice:"Lo popular es la historia de lo excluido: de los que no tienen patrimonio o no logran que ese patrimonio sea reconocido y conservado..." (Zubieta, 2004, citado en Lacarrieu, 2006). Y los excluidos o carentes de patrimonio han sido colocados históricamente en las "aldeas de los nativos", en los espacios del "buen salvaje", imposibilitados de reconocimiento y legitimación de su cultura." (Lacarrieu, 2006).

Tomando en cuenta estos aportes, entendemos por patrimonio las prácticas y discursos de las poblaciones locales que no necesariamente son aquellas institucionalizadas por los poderes políticos o sociales hegemónicos: las prácticas productivas locales, fiestas y tradiciones, las formas de hacer que tienen especial significancia para quienes las utilizan. Todo ello puede constituirse en patrimonio, si los pobladores lo reconocen y significan positivamente.

A pesar de ser una zona con valor histórico y productivo para la ciudad de La Plata, el territorio en el que se lleva adelante nuestro proyecto, no es reconocido por esas características ni tampoco valorado por la propia comunidad ni por el sector turístico. Es una zona productiva que en los últimos 20 años ha crecido en forma exponencial, llegando a considerarse como uno de los principales cinturones productivos de la Provincia de Buenos Aires, y del país. La población que trabaja en el sector flori-hortícola es hoy y ha sido históricamente, en su mayoría de origen migrante, lo que genera en este territorio gran diversidad cultural así como complejidad en las dinámicas sociales.

Abordar el territorio desde la Universidad, junto a la población local, desde una mirada patrimonial, genera nuevos diálogos en los que este concepto y sus múltiples definiciones entran en tensión entre sí y con los sentidos comunes que circulan socialmente -que constantemente ponemos en juego-. Llegar al reconocimiento y valoración de los propios esquemas culturales por parte de la población local conlleva dificultades en poblaciones o áreas subalternas, pero las dificultades no están sólo allí, sino también en producir discusiones hacia adentro del equipo extensionista sobre estos conceptos, que desde algunas teorías se muestran como monolíticos y de significados unívocos. De esta forma se ten- 
san y reconfiguran los conocimientos adquiridos en la teoría, generando nuevas formas de comprender a la comunidad y con la comunidad.

\begin{abstract}
Llegar al reconocimiento y valoración de los propios esquemas culturales por parte de la población local conlleva dificultades en poblaciones 0 áreas subalternas, pero las dificultades no están sólo allí, sino también en producir discusiones hacia adentro del equipo extensionista sobre estos conceptos, que desde algunas teorías se muestran como monolíticos y de significados unívocos. De esta forma se tensan y reconfiguran los conocimientos adquiridos en la teoría, generando nuevas formas de comprender a la comunidad y con la comunidad.
\end{abstract}

Por otro lado, al trabajar con productores locales (florícolas, hortícolas) y con emprendedores de la localidad, nos encontramos promoviendo lo que se denomina Economía social y solidaria. La ESS se configura como un ámbito donde los conocimientos o saberes individuales y sociales, las construcciones asociativas y otros recursos intangibles como la identidad, los valores y la confianza, se constituyen como "reservas ocultas". En este sentido la Economía Social (ES) ayuda a construir tramas socio-productivas sustentables en las que el esfuerzo y la energía social, articulados con los intereses individuales, son capitalizados en favor del bien común. Dicha construcción implica cuestionar las lógicas duales de inclusión-exclusión y apuntar a sociedades más equitativas e igualitarias (Arroyo, 2006). La ESS es un campo teórico en permanente revisión a través de las prácticas territoriales, donde existen diferentes propuestas que buscan formas de producir, comerciar y consumir más justas, equitativas, solidarias, basadas en relaciones sociales de respeto mutuo, autogestivas y pro-ambientales.

En esta relación de la extensión universitaria y la ES, Maidana plantea que“.. .la extensión es una herramienta fundamental para que la práctica universitaria se profundice como dinámica de acción y reflexión. A partir de su vinculación con la investigación y la docencia podrá aumentar su contribución a los debates dentro y fuera de la universidad, convirtiéndose en un aporte central para el desarrollo de la Economía Social." (2014)

Precisamente la extensión debe contribuir a que la universidad se reconfigure como recurso activo, lúcido y crítico de los procesos transformadores de la economía y de la sociedad² (Maidana, 2014). Desde

2 El autor hace referencia al término activo, a la producción de iniciativas desde una perspectiva propia, en diálogo con la docencia y en diálogo con la investigación; a término lúcido: a que la práctica universitaria no debe ser pura aplicación de conceptos y teorías desarrolladas por otros, ya que la práctica es el escenario ideal de producción y re-creación de los conceptos y la criticidad como uno de los núcleos centrales de la autonomía universitaria. (Maidana, 2014) 
esta perspectiva, en el territorio se han implementado diferentes estrategias para jerarquizar la producción local de la agricultura familiar y la práctica artesanal en las comunidades que integran el periurbano platense, y para revalorizar las identidades y culturas locales. Consideramos que la ESS constituye el camino y el horizonte a alcanzar, donde la agricultura familiar y los emprendimientos productivos individuales logren enmarcarse, acercándose a los valores que implican este paradigma: solidaridad, precio justo, cuidado de la naturaleza, calidad de las relaciones de trabajo, integración social y acceso a derechos ciudadanos, centralidad del trabajo colectivo, asociado y autogestionado.

En este sentido, la extensión universitaria hizo aportes importantes al desarrollo de la ES. Por un lado, su contribución a los debates dentro y fuera de la universidad y del sistema universitario en general; por otro, propiciar la vigencia del pensamiento crítico, dado que en la práctica suele producirse la capitulación de los principios; finalmente, en muchas oportunidades la ES ha llegado a la universidad por la puerta de la extensión, causando que estas prácticas surgidas en el territorio hayan interpelado a la universidad para pensar críticamente los nuevos procesos y actores socioeconómicos. (Maidana, 2014)

Retomando los procesos territoriales, uno de los grandes problemas a los que se enfrentan, tanto las casas de altos estudios como las y los integrantes de la comunidad universitaria, a la hora de fijar objetivos en relación a la vinculación con la comunidad en general, es la ausencia de herramientas que articulen las relaciones complejas que existen entre estos dos ámbitos y la escasa formación en extensión universitaria hacia adentro de la institución educativa, cuestión que se ha abordado en forma creciente en los últimos años.

La falta de interpretación del territorio, sus actores político-sociales, las barreras idiosincráticas, las costumbres ancestrales, el sistema de jerarquías internas de los diferentes grupos e instituciones, entre tantos otros factores, son importantes trabas que influyen en el éxito de una estrategia de extensión fabricada puertas adentro de los claustros universitarios. En este sentido, al iniciar el trabajo en territorio en 2012, notamos que no había articulación de la UNLP con las instituciones locales de la zona abordada3. Como consecuencia directa de esta situación, fue arduo el trabajo del equipo para poder insertarse en la comunidad donde se iba a llevar adelante el proyecto en cuestión. El camino recorrido en las primeras etapas sirvió de experiencia para reflexionar sobre la necesidad de buscar "porteros" adecuados en el territorio, que faciliten el establecimiento y el desarrollo de las actividades planificadas.

Posteriormente, la vinculación con el CCEU N ${ }^{\circ} 5$ de Abasto de la UNLP, permitió articular con diversas instituciones locales, entre ellas

3 El proyecto se inicia en Colonia Urquiza, perteneciente a la localidad de Melchor Romero, articulando con las escuelas y ONG de la zona. 
las instituciones educativas. Si bien las actividades en las comunidades educativas probaron tener un amplio y positivo impacto, aún persistía el desafío de trascender la limitación de estas instituciones para desarrollar acciones en territorio de puertas afuera con las comunidades locales. Esta limitación fue superada por una estrategia de convocatoria personalizada a actores claves territoriales y comunitarios con objetivos y propuestas concretas, que generó la constitución de la "Mesa Intersectorial de Abasto". Debe destacarse que esta estrategia también fue posible por la mediación del Consejo Social de la UNLP que permitió establecer primeros contactos con las y los actores territoriales del ámbito productivo.

La mesa intersectorial, como estrategia de extensión, se estableció como un espacio de encuentro y de diálogo, construido de manera horizontal entre las y los diferentes actores y sectores del periurbano platense quienes aportan sobre las necesidades y problemáticas a abordar. El funcionamiento de la misma se enmarcó en los conceptos y valores de la ESS mencionados anteriormente. El producto de esta estrategia se plasmó en actividades en las que la ESS se lleva a la práctica. Estos son "Encuentros de productores y consumidores" y la feria "El Paseo de productores en Abasto" como espacios de intercambio de saberes y experiencias, valorización de la zona productiva y las y los productores, y comercialización de productos frescos a precio justo.

Otra estrategia de extensión utilizada fue la planificación conjunta y desarrollo de recorridos turístico-recreativos, con visitas a quintas de productores flori-hortícolas de la agricultura familiar y viveristas, que tuvieron como propósito dar la palabra a las y los productoras, y que se constituyan como sus propios representantes ante otros grupos sociales, de esta manera valorizar el patrimonio productivo, cultural y ambiental de la zona, priorizando principios y valores basados en trabajo colaborativo y los saberes de cada comunidad. Ambas estrategias posibilitaron el acercamiento y reconocimiento de las y los visitantes-consumidores hacia las y los productores, demostrando su interés por los procesos productivos y la preocupación por la situación socio-económica que padecen los productores y productoras de la agricultura familiar, ampliando y diversificando de esta forma las y los destinatarios de los proyectos, que no se resumen a las y los productores como destinatarios directos, sino que incluyen a las y los consumidores como destinatarios indirectos y como actor principal de la relación dialéctica del proceso productivo.

\section{DE LOS PROCESOS DE CONSTRUCCIÓN DEL CONOCIMIENTO Y DE ENSEÑANZA Y APRENDIZAJE}

Las prácticas en territorio habilitan a las y los docentes y estudiantes/extensionistas el contacto directo con las problemáticas de las y los productores y sus saberes, al tiempo que los conocimientos y 
concepciones que traen consigo las y los sujetos-otredad y las organizaciones sociales locales, nos permiten conocer las formas de habitar el territorio con el que trabajamos. La ocurrencia de estos dos acontecimientos, de la mano de una mirada curiosa, respetuosa y comprensiva, viabiliza la construcción conjunta y apropiación de nuevos conocimientos, basados en el diálogo de experiencias académicas y populares. Dicha conjunción es la base central para la construcción de conocimientos críticos y situados, donde se expresen los problemas y posibles soluciones en el territorio, donde las y los sujetos sociales locales no son fuente primaria de información, sino protagonistas y agentes activos de la identificación de problemas y de la construcción de soluciones para transformar las problemáticas territoriales.

Las prácticas en territorio habilitan a las y los docentes y estudiantes/extensionistas el contacto directo con las problemáticas de las y los productores y sus saberes, al tiempo que los conocimientos y concepciones que traen consigo las y los sujetos-otredad y las organizaciones sociales locales, nos permiten conocer las formas de habitar el territorio con el que trabajamos. La ocurrencia de estos dos acontecimientos, de la mano de una mirada curiosa, respetuosa y comprensiva, viabiliza la construcción conjunta y apropiación de nuevos conocimientos, basados en el diálogo de experiencias académicas y populares.

Entonces y a modo de reflexión, nos preguntamos de qué manera se relacionan las lógicas propias universitarias y las lógicas de las y los actores territoriales que posibilitan una co-construcción del conocimiento sobre las problemáticas sociales locales, sobre las propias prácticas universitarias, que transforman las políticas y estructuras de la universidad misma. El conocimiento producido se convierte en insumo para la resolución de problemas de ambos sectores. Además, dicha práctica transforma la realidad territorial, las relaciones de la universidad con el territorio y de las y los actores que toman parte en ella, al tiempo que enriquecen la mirada de los distintos sujetos sociales, teniendo como eje y objetivo el reconocimiento del otro, su experiencia, y su lógica. (Giraudo y Vergerio, 2017)

En este contexto, Boaventura de Sousa Santos (2006) en su obra "Sociología de las ausencias" plantea que la universidad debe asumir la radicalización de la democratización del conocimiento, realizando lo que denomina "una extensión al revés": atrayendo al interior de la universidad los saberes populares. La democratización no es sólo un proceso de circulación de conocimientos, es también producción de 
los mismos, visibilización y legitimación, de este modo se materializa la voz de las y los actores sociales locales. Sobre este concepto, desde la práctica extensionista, nos planteamos como desafío la visibilidad de los saberes propios de los productores flori-hortícolas y emprendedores, sus procesos productivos y sus productos. Las y los productores son invisibilizados y discriminados por su condición migrante (interna o externa) profundizados en los últimos años por las políticas discriminatorias y xenófobas implementadas.

Asimismo, planteamos la posibilidad de generar conocimiento científico a partir de la extensión universitaria, de la práctica investigativa comprometida y no extractivista, que construye con otros y otras, dándole voz y entidad al saber popular. Buscando reflexionar sobre lo que de Sousa Santos (2006), llama la monocultura del saber y del rigor, que supone que la única forma de saber riguroso es la que se produce en los ámbitos científicos, desestimando de esta forma los conocimientos que se producen en otros ámbitos sociales: las prácticas sociales que están basadas en conocimientos populares, conocimientos indígenas, conocimientos campesinos, conocimientos urbanos, que no son evaluados como importantes o rigurosos. De esta forma, el conocimiento científico "al constituirse como monocultura (como la soja), destruye otros conocimientos, produce lo que llamó "epistemicidio": la muerte de conocimientos alternativos. Reduce la realidad porque "descredibiliza" no solamente a los conocimientos alternativos sino también a los pueblos, los grupos sociales cuyas prácticas son construidas en esos conocimientos alternativos". (de Sousa Santos, 2006: 24)

\section{planteamos la posibilidad de generar conocimiento científico a partir de la extensión universitaria, de la práctica investi- gativa comprometida y no extractivista, que construye con otros y otras, dándole voz y entidad al saber popular.}

Coincidimos con la premisa que sostiene la necesaria relación permanente entre la universidad y el contexto social para que la universidad ponga en juego su función social (Giraudo y Vergerio (2017). Desde esta perspectiva y con el apoyo y acompañamiento de la UNLP, desarrollamos una serie de acciones que propendieron al acercamiento de las y los consumidores y productores a través del diálogo de saberes y experiencias que visibilizaron las problemáticas sociales, económicos, culturales y ambientales de las y los pobladores en general y las y los productores en particular. A la vez, dichas acciones permitieron la construcción conjunta y democratización de nuevos conocimientos allí generados. Todo proceso de intervención puede o 
debe ser a su vez proceso de construcción del conocimiento como así también de enseñanza. Así, la extensión universitaria se constituye en una función estratégica, en tanto su capacidad de articular la docencia con la investigación, con la preservación y difusión de la cultura y, de esta manera, favorecer la formación integral de las y los estudiantes y demás integrantes de la comunidad universitaria orientado a la formación de individuos reflexivos y comprometidos con una sociedad más justa. (Orozco, 2004)

En la misma perspectiva Humberto Tommasino (2010:2) plantea que "En las prácticas integrales, en donde se articulan las funciones, el rol de la extensión puede propiciar la reconfiguración, integración y renovación de todos los componentes del acto educativo" y agrega que "...las experiencias educativas en terreno posibilitan procesos que, a la vez, interpelan los conocimientos adquiridos en las aulas y posibilitan su mejor internalización, al vincular a las y los estudiantes con problemáticas sociales de su tiempo y ponerlos a trabajar junto a las comunidades que las viven, promueven procesos de transformación subjetiva y reflexión ético-crítica favorecedoras de la asunción de posicionamientos personales y colectivos críticos, responsables y solidarios".

Por otro lado, los procesos de enseñanza y aprendizaje contextuados en las prácticas de la extensión universitaria suponen trabajar desde la perspectiva de la complejidad. Esto implica, según García (2004), una transición de la visión simple a la compleja que supone la construcción de la idea de interacción, entendiendo al mundo como un conjunto de sistemas que requiere que se comprenda que las interacciones existentes entre los elementos componentes del medio son las que organizan esos sistemas.

La inmersión en diferentes ámbitos del territorio conlleva un aprendizaje sobre las lógicas de las y los distintos actores sociales, sus intereses y el contexto en el cual se dan, o sea de variables que interaccionan. La dificultad está dada por la imposibilidad de apartar variables de situaciones sociales reales, para poder comprender mejor sólo algunos aspectos de las mismas. Los intereses, los juicios y prejuicios, los miedos, la toma de posición, las simpatías, y muchas otras variables actúan en un mismo tiempo y espacio, provocando constantes aperturas y cierres para la comprensión y la continuidad de la tarea propuesta. El diálogo con ese "sujeto-otredad", la interpelación, la escucha, la construcción conjunta llevan al replanteo constante de las intervenciones en territorio. La práctica de extensión pone en constante tensión los conocimientos con los que creemos "contar" y habilita la reflexión acerca de lo que sucede, por qué acontece de esta forma, y con qué herramientas conceptuales interpretamos esa porción de realidad, cuáles nos ayudan a repensar las vivencias y cuales necesitamos transformar, conformándose así en una fuente de aprendizaje. 
El diálogo con ese "sujeto-otredad", la interpelación, la escucha, la construcción conjunta llevan al replanteo constante de las intervenciones en territorio. La práctica de extensión pone en constante tensión los conocimientos con los que creemos "contar" y habilita la reflexión acerca de lo que sucede, por qué acontece de esta forma, y con qué herramientas conceptuales interpretamos esa porción de realidad, cuáles nos ayudan a repensar las vivencias y cuales necesitamos transformar, conformándose así en una fuente de aprendizaje.

En el abordaje de la realidad social, contexto y corazón de la extensión universitaria, convergen entonces intereses y variables de naturaleza compleja, donde interactúan elementos y sistemas del mundo físico con dimensiones de lo social mezcladas con intereses económicos, modelos científicos, estructuras políticas, representaciones simbólicas y culturales que atentan permanentemente los límites artificiales de los campos del saber y sus lógicas de funcionamiento, sus métodos de trabajo, sus estándares de legitimación y sus patrones de utilidad (Garcia, 2006). En este contexto, de constante cambio y tensión, se generan múltiples aprendizajes para el grupo: se aprehende la complejidad de la realidad en la cual se quiere incidir, se ponen a prueba los marcos conceptuales a través de los cuales se lee el territorio, se transforma y consolida el equipo de trabajo, y se forjan aptitudes personales en las y los integrantes.

\section{PALABRAS FINALES}

El modo de interacción de la UNLP con su entorno, que refleja el trabajo realizado desde el proyecto de extensión como experiencia particular, representa una relación de ida y vuelta en la que la extensión abandona el tradicional perfil asistencialista y transferencista, para pasar a construir la agenda de trabajo con la propia comunidad, siendo vehiculizador de ideas y articulador de distintos sectores. Consideramos que esta es la manera en que la extensión define su rol social en la actualidad, como un actor estratégico, crítico y reflexivo del y en el contexto.

La vinculación de la universidad con la sociedad es un trabajo que interpela constantemente nuestro lugar de "universitarias" $y$ "universitarios". La posibilidad del trabajo conjunto, horizontal, democrático y sostenido en el tiempo son las herramientas fundamentales para comprender el "hacer" de la extensión. 
La vinculación de la universidad con la sociedad es un trabajo que interpela constantemente nuestro lugar de "universitarias" y"universitarios". La posibilidad del trabajo conjunto, horizontal, democrático y sostenido en el tiempo son las herramientas fundamentales para comprender el "hacer" de la extensión.

Asimismo, consideramos que el proceso de co-construcción y democratización del conocimiento, es el medio para el trabajo compartido con los sectores populares, para el desarrollo de procesos de enseñanza y aprendizaje de estudiantes, docentes e integrantes de las comunidades locales y un acercamiento certero de nuestro espacio universitario al territorio. Las reflexiones devenidas del reconocimiento de múltiples trayectorias y saberes, y de la necesidad de construir conocimientos "con otras y otros" y no "sobre otras y otros", debe ser el antídoto a la "autoridad académica" per se, y al histórico divorcio entre la investigación y la extensión universitaria, que las ve como actividades contrapuestas y de distinta jerarquía.

De esta manera y retomando a de Sousa Santos, la práctica universitaria está transformando las "ausencias" en emergentes visibles, válidos y creíbles a través de un nuevo modo de producción de conocimiento donde el saber académico pueda dialogar con el saber popular.

La extensión universitaria, en su dimensión de acción territorial, con la necesidad de reconocimiento de actores sociales relevantes y problemáticas principales, en la búsqueda de los conocimientos e interpretaciones locales sobre dichas cuestiones, necesariamente realiza diagnóstico, elabora hipótesis, construye y reflexiona sobre alternativas de abordaje y llega a conclusiones, siempre provisorias en tanto conocimientos situados. De este modo los procedimientos de la extensión universitaria no difieren de los de la investigación. La distancia entre estas actividades se da principalmente en que en la extensión los procesos no son artificialmente limitados (ni en tiempo, ni en espacio, ni en variables) y el conocimiento no es sólo académicamente producido, sino que es constantemente elaborado, corregido y reelaborado entre las y los múltiples actores actuantes. De esta forma, el conocimiento no sólo se produce de una forma más democrática, sino que sobre él se ejercen más controles, permitiendo que haya menos sesgos académicos y sectoriales.

En cuanto a las estrategias de extensión implementadas, como se señaló en el desarrollo del artículo, se apuntó a establecer relaciones más equitativas y democráticas entre productores y consumidores, que además formaron parte de la construcción de otra economía. Los principios de la ESS permitieron abordar y problematizar significados naturalizados tanto entre las y los productores y consumidores, como 
en el propio equipo extensionista. La Mesa Intersectorial resultó una herramienta fundamental para el trabajo del proyecto, para la reflexión conjunta y la progresiva organización de los encuentros y ferias, cuyo objetivo no fue sólo económico, sino también social y cultural.

Trabajar desde la extensión, la práctica universitaria, implica integrar el conjunto de procesos descriptos, que articulan la extensión, la docencia y la investigación. 


\section{BIBLIOGRAFIA}

Arroyo, Daniel. (2006). "La Economía Social como estrategia de inclusión", FLACSO, Argentina. 6-17.

Bertoncello R. (2002). "Turismo y Territorio. Otras miradas". Aportes y Transferencias, Mar del Plata, 6 (2) 31-50

Camilloni, A. (2017). “El desarrollo de las multialfabetizaciones en las experiencias de extensión". Revista +E versión en línea, 7(7),60-67. Santa Fe, Argentina: Ediciones UNL. Recuperado de https://bibliotecavirtual.unl.edu.ar/publicaciones/ index.php/Extension/article/view/7051

Carli, S. (2008). "Manifiesto Liminar de la Reforma Universitaria" (Federación Universitaria de Córdoba, 1918). Transatlántica de educación, (5), 35-42. Recuperado de https://dialnet.unirioja.es/servlet/articulo?codigo=3036611

De Sousa Santos, Boaventura. (2006). “Capítulo I. La Sociología de las Ausencias y la Sociología de las Emergencias: para una ecología de saberes". En publicación: Renovar la teoría crítica y reinventar la emancipación social (encuentros en Buenos Aires). Recuperado de http://www.bibliotecavirtual.clacso.org. ar/ar/libros/edicion/santos/Capitulo\%20l.pdf

Drago, N. (2016). "Extensión universitaria y economía social. El Paseo de Economía Social y Solidaria de la UNLP". Tesis de grado. Universidad Nacional de La Plata. Facultad de Humanidades y Ciencias de la Educación. Recuperado de http://www.memoria.fahce.unlp.edu.ar/tesis/te.1227/te.1227.pdf

Gallopin, G. (2003). "Sostenibilidad y Desarrollo sostenible. Un enfoque sistémico". Serie Medio Ambiente y Desarrollo. CEPAL, ONU Chile. N64, 41. Recuperado de https://repositorio.cepal.org/handle/11362/5763

García Canclini, N. (1999). “Los usos sociales del patrimonio cultural". En: Aguilar Criado, Encarnación (Ed.), Patrimonio etnológico. Nuevas perspectivas de estudio (pp. 6- 33). Consejería de la cultura. Junta de Andalucía. Recuperado de http:// observatoriocultural.udgvirtual.udg.mx/repositorio/handle/123456789/130

García, J. E. (2004). "Educación ambiental, constructivismo y complejidad". Sevilla, Diada, pp. 219.

García, R. (2006). "Sistemas complejos. Conceptos, método y fundamentación epistemológica de la investigación interdisciplinaria". 1 º ed. Gedisa, Barcelona, pp. 201. Recuperado de http://www.proglocode.unam.mx/contenido/ ii-aspectos-metodologicos

Giraudo C. y Vergerio G. (2017). “La extensión universitaria: en busca del saber, del conocimiento colectivo y la construcción de la demanda". Revista Ext. Divulgación y discusión referidas a la extensión universitaria. Universidad 
de Córdoba. Argentina. Recupera de https://revistas.unc.edu.ar/index.php/ ext/article/view/14466

Gómez S., Rossi E., Marquez G. J., Molinari G., Rampello P. y Mallo J. (2014). "Aciertos y desaciertos de una experiencia en territorio. Evaluación del proceso de patrimonialización realizado en la zona periurbana de la ciudad de La Plata". 1 ras Jornadas sobre reflexión sobre la praxis en extensión de la FCNyM-UNLP. La Plata. Buenos Aires. Argentina.

Gomez S. y Marquez G. (2015). “Evaluación del proceso de patrimonialización realizado en la zona periurbana de la ciudad de La Plata". XI Jornada Nacional de Extensión Universitaria - III Jornadas Regionales Metropolitanas. Lanús. Buenos Aires. Argentina. Recuperado de https://www.researchgate.net/publication/326835167_Evaluacion_del_proceso_de_patrimonializacion_realizado_en_la_zona_periurbana_de_la_ciudad_de_La_Plata

Gomez S., Rossi E., Rampello P., Marquez G. y Molinari G. (2016). “La construcción del patrimonio local como forma de intervención para descotidianizar el territorio". 3ras Jornadas Latinoamericanas de Patrimonio y Desarrollo. La Plata. Buenos Aires. Argentina. Recuperado de https://www.aacademica. org/silvina.gomez/25

Maidana, D. (2014). "La extensión universitaria y la economía social. Voces en el Fénix. La estrategia del caracol. Revista del Plan Fenix. Año 5 №37, 138146. Recuperado de https://www.vocesenelfenix.com/content/la-extensi\%C3\%B3n-universitaria-y-la-econom\%C3\%ADa-social

Frezán Orozco, M. (2004). “La extensión universitaria y la universidad pública". Revista Reencuentro. México DF, №39. Recuperado de https://reencuentro.xoc.uam.mx/index.php/reencuentro/article/view/477

Pastore, R. (2015). “Las universidades públicas argentinas y la Economía Social y Solidaria. Hacia una educación democrática y emancipadora". En Revista +E versión digital, (5), 20-31. Santa Fe, Argentina: Ediciones UNL. Recuperado de https://bibliotecavirtual.unl.edu.ar/publicaciones/index.php/Extension/article/view/5139

Rodríguez R. J. (2010). “Elementos para el desarrollo de una teoría territorial". UAM

Rossi E., Gómez S., Rampello P., Molinari G., Márquez G. J., Mallo J., Bravo G., Álvarez L., García C., Taus Y., Asarchuk V., Rosiere D. y Álvarez M. (2016a). “Patrimonialización y Turismo: proyecto de extensión universitaria". II Jornada de Agricultura Familiar en Naturales. La Plata. Buenos Aires. Argentina.

Rossi E., Gomez S., Marquez G., Rampello P. y Molinari G. (2016b). "El turismo como articulador social, cultura y productivo". VII Congreso Nacional de Extensión Universitaria. Paraná, Entre Ríos. Argentina.doi: 10.13140/RG.2.2.19474.30404 